\title{
ACTIVITÉ COCCIDIOSTATIQUE DES ASSOCIATIONS FURAZOLIDONE - NITROFURAZONE
}

\author{
PAR \\ J. AYCARDI \\ Station de Recherches avicoles (.. N.R.Z., Jony-en-Josas (S. et O.).
}

\section{SOMMAIRE}

Une expérimentation portant sur 300 poulets de chair inoculés de coccidiose cæcale, et traités préventivement avec des associasions furazolidonenitrofurazone en addition continue dans la ration, indique que la meilleure protection contre le développement des parasites est obtenue avec le mélange $50 \mathrm{~g} / 50 \mathrm{~g} \mathrm{p}$. tonne des deux produits. Cette association est donc préférable à celle qui est couramment employée (furazolidone $8 \mathrm{~g}+$ nitrofurazone $55 \mathrm{~g}$ p. tonne). L'amélioration de la croissance et de l'indice de consommation confirme les données relatives à l'activité antiparasitaire. La furazolidone seule à Ioo $\mathrm{g} \mathrm{p}$. tonne, tout en exerçant une inhibition moindre du parasitisme, permet néanmoins, en milieu contaminé, l'obtention de gains de poids supérieurs à tous les autres traitements. Les divers coccidiostats n'entravent pas l'installation de l'immunité anticoccidienne.

L'emploi des associations furazolidone - nitrofurazone comme anticoccidien préventif chez les volailles est aujourd'hui répandu. Cependant certaines irrégularités d'action enregistrées sur le terrain, posaient la question de savoir si le mélange couramment utilisé : furazolidone $8 \mathrm{~g}$ + nitrofurazone $55 \mathrm{~g}$ par tonne d'aliment, représentait l'association optimale. Aucune donnée de cet ordre n'existant dans la littérature, nous avons tenté une expérimentation sur 300 poussins type Vilvert argentés répartis en 6 lots selon le tableau $\mathrm{I}$. 
TABLEAU I

\begin{tabular}{|c|c|c|c|c|c|}
\hline Lot & Effectif & Code & & & Traitement \\
\hline I & $5^{\circ}$ & $\mathrm{CFZ} 8 / 55$ & + & + & $\begin{array}{l}\text { Furazolidone } 8 \mathrm{~g} / \text { tonne } \\
\text { Nitrofurazone } 55 \mathrm{~g} / \text { tonne }\end{array}$ \\
\hline 2 & 50 & $\mathrm{CFZ}{ }_{15} / 55$ & + & + & $\begin{array}{l}\text { Furazolidone } 5 \mathrm{gg} / \text { tonne } \\
\text { Nitrofurazone } 50 \mathrm{~g} / \text { tonne }\end{array}$ \\
\hline 3 & 50 & $\mathrm{CFZ}_{50 / 50}$ & + & + & $\begin{array}{l}\text { Furazolidone } 5 \circ \mathrm{g} / \text { tonne } \\
\text { Nitrofurazone } 5 \mathrm{gg} / \text { tonne }\end{array}$ \\
\hline $\begin{array}{l}4 \\
5 \\
6\end{array}$ & $\begin{array}{l}5^{\circ} \\
5^{\circ} \\
50\end{array}$ & $\begin{array}{l}\mathrm{CF} \text { Ioo } \\
\text { CT } \\
\text { ST }\end{array}$ & + & $\begin{array}{l}+ \\
+ \\
t\end{array}$ & $\begin{array}{c}\text { Furazolidone roog/tonne } \\
\text { Néant } \\
\text { Néant }\end{array}$ \\
\hline
\end{tabular}

L es poussins, élevés en batterie, ont été inoculés de la façon suivante:

I re série : I ooo ookystes sporulés (Eimeria tenella) au $\mathrm{I}^{\mathrm{e}}$ jour, 5000 ookystes sporulés au $\mathrm{I} 8 \mathrm{e}$ jour et 40 o00 ookystes sporulés au I $9^{\mathrm{e}}$ jour, correspondant à l'épreuve d'Effficacité.

$2^{\mathrm{e}}$ série : 50000 ookystes sporulés (Eimeria tenella) au $5^{6^{\mathrm{e}}}$ jour, correspondant à l'épreuve d'Immunité.

Les aliments supplémentés ont été distribués du $3^{\mathbf{e}}$ jour au $84^{\mathrm{e}}$ jour (I2 semaines). Pendant les deux premiers jours, tous les lots ont été alimentés au maïs.

Les obervations et mesures ont porté sur la mortalité, la croissance (pesée individuelle), l'élimination quotidienne d'ookystes par animal du jour 15 au jour 38 et du jour 55 au jour 79 .

Au cours de l'épreuve d'Efficacité (Fig. I) tous les lots traités éliminent beaucoup moins d'ookystes que les témoins. Il y a d'importantes variations selon les dosages. La meilleure protection correspond à l'association 50/50 Furazolidone/ Nitrofurazone. Les données relatives à 1a mortalité et aux gains de poids sont rassemblées dans le tableau II, ainsi que les indices de consommation à 56 et 84 jours. Dans tous les lots traités, les gains de poids sont constamment supérieures aux témoins, et la mortalité significativement plus faible.

Les courbes d'élimination d'ookystes au cours de la seconde épreuve (persistance de l'Immunité acquise à la suite de la première inoculation) montrent que les lots traités ont une immunité du même ordre que le témoin CT (Fig. II). Le lot 3 possède une immunité nettement supérieure à tous les autres lots. Les lots 2,3 et 4 subissent une mortalité inférieure à celle des témoins naturellement imunisés. Quant aux gains de poids (tableau II), seuls les lots 3 et 4 ont une supériorité significative. Ce sont aussi les lots où les indices de consommation à 84 jours sont significativement abaissés par rapport aux lots 5 et 6 . Poids à I 2 semaines et mortalités cumulées donnent des résultats analogues. 


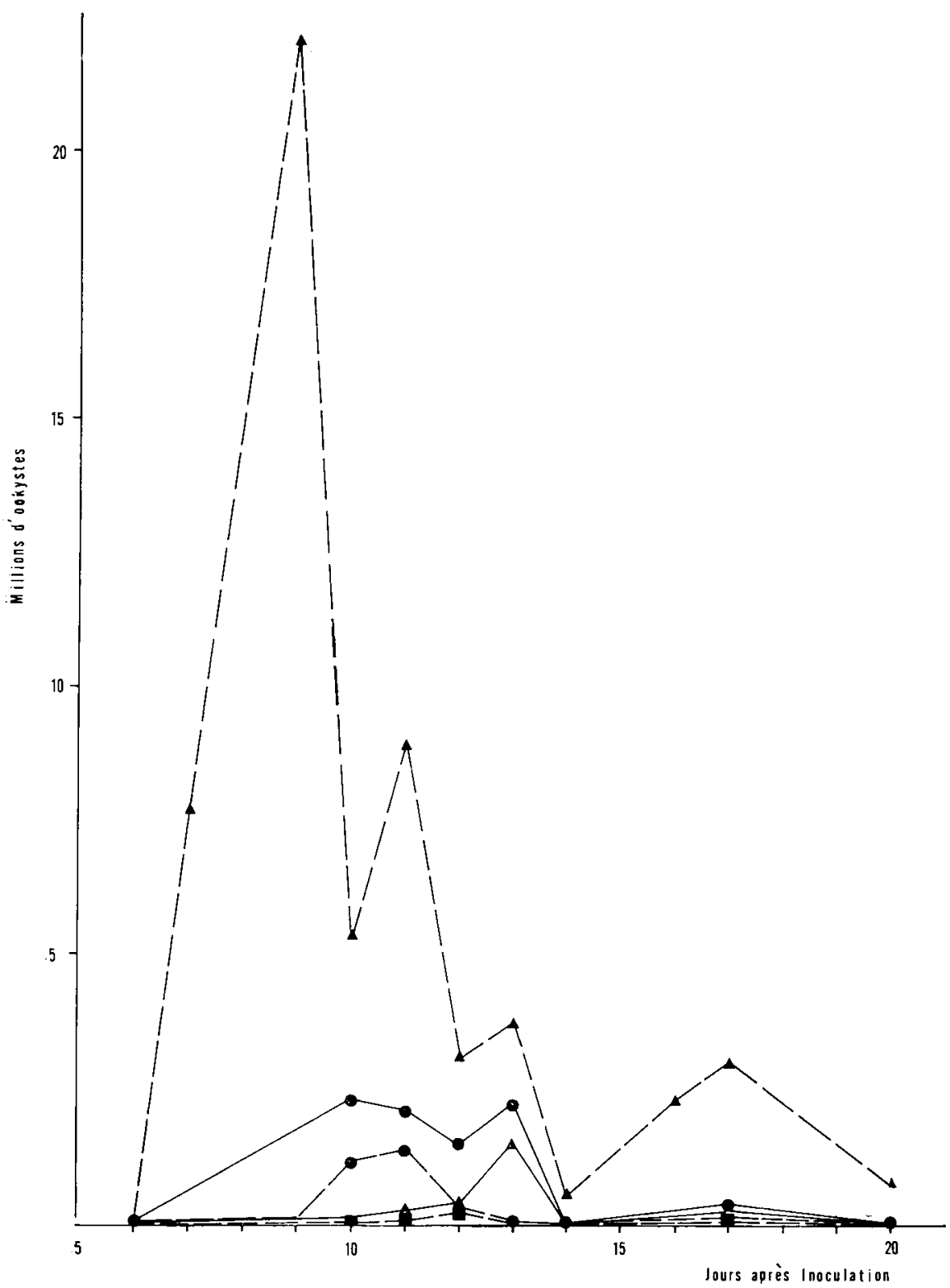

FIG. I. - Elimination quotidienne d'ookỵtes par animal dans les fécès.

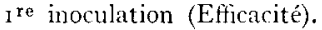

Abréviations identiques an tableau $\mathrm{I}$.

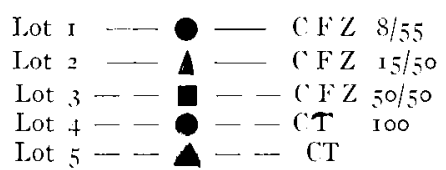




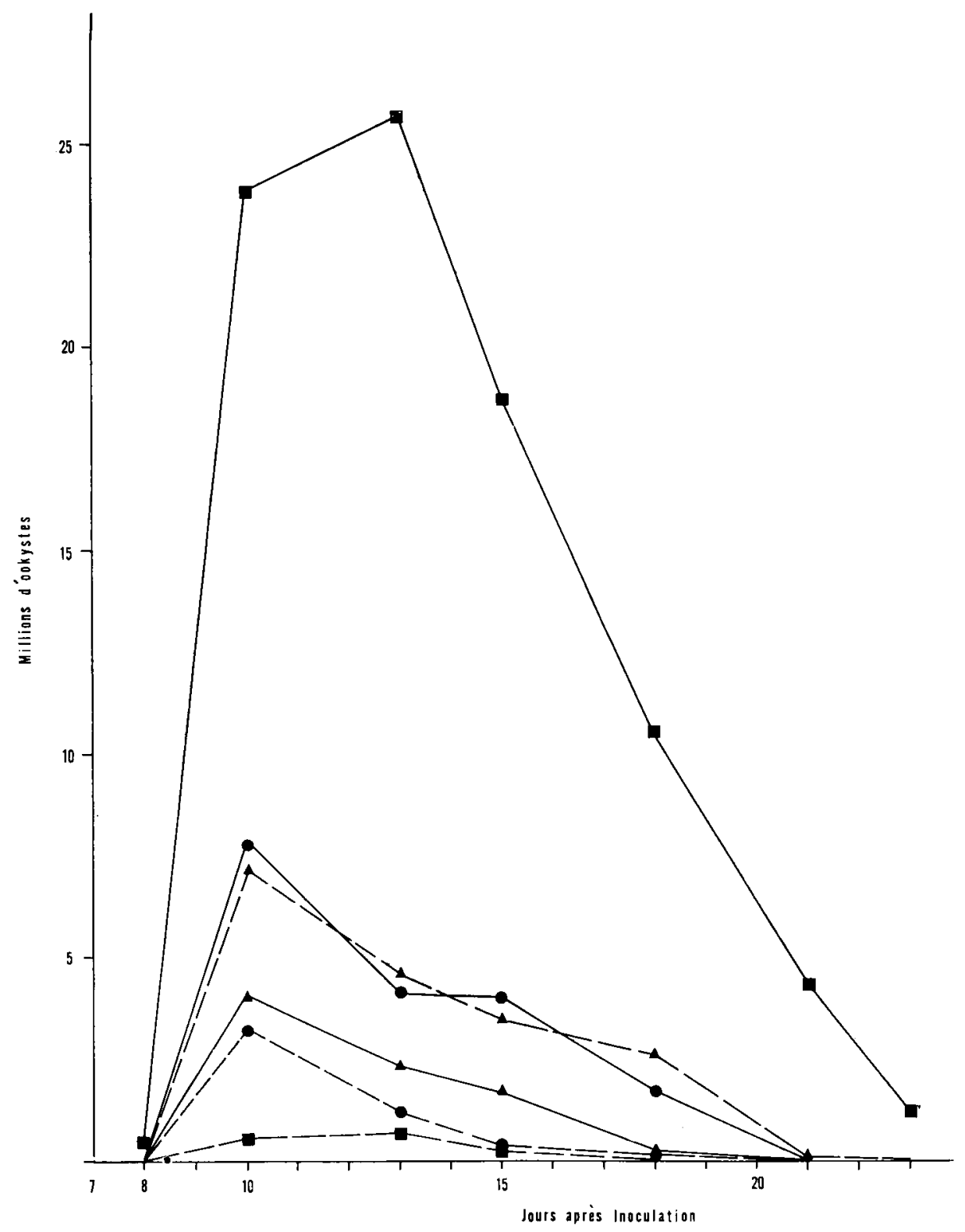

FIg. 2. - Klimination quotidienne d'ookystes par animal dans les fécés. $2^{\mathrm{e}}$ inoculation (Immunité).

Abréviations identiques au tableau I.

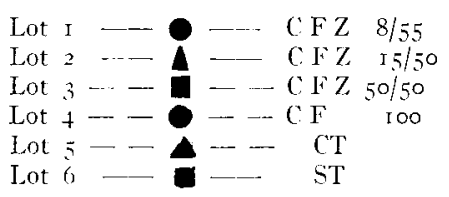


TABLEAU II

\begin{tabular}{|c|c|c|c|c|c|c|}
\hline LOTS & $\mathrm{CFZ}^{\mathbf{1}} 8 / 55 \mid$ & $\mathrm{CFZ}_{15 / 50}^{2}$ & $\mathrm{CFZ}_{5}^{3} / \mathrm{O}^{\circ}$ & $\mathrm{CF}_{100}^{+}$ & cT & $\begin{array}{l}6 \\
\text { ST }\end{array}$ \\
\hline $\begin{array}{l}\text { Ire Inoculation. Efficacité. } \\
\text { Période du } \mathrm{I} 7^{\mathbf{e}} \text { au } 5^{\mathbf{e}} \text { jour. } \\
\text { Gain de poids en g. } \ldots \ldots \ldots \\
\mathrm{Kg} \text { aliment } / \mathrm{Kg} \text { poids vif... } \\
\text { Mortalité p. roo........... }\end{array}$ & $\begin{array}{c}564 \\
2,30 \\
6\end{array}$ & $\begin{array}{c}5+1 \\
2,4 I \\
4\end{array}$ & $\begin{array}{c}554 \\
2,38 \\
4\end{array}$ & $\begin{array}{c}574 \\
2,32 \\
4\end{array}$ & $\begin{array}{c}527 \\
2,95 \\
\mathrm{I} 4\end{array}$ & $\begin{array}{c}55^{6} \\
2,34 \\
0\end{array}$ \\
\hline 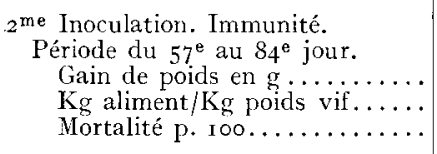 & $\begin{array}{c}5 \text { I0 } \\
3,26 \\
8\end{array}$ & $\begin{array}{c}535 \\
2,20 \\
4\end{array}$ & $\begin{array}{c}614 \\
2,98 \\
2\end{array}$ & $\begin{array}{c}629 \\
2,77 \\
2\end{array}$ & $\begin{array}{c}530 \\
4,1,3 \\
10\end{array}$ & $\begin{array}{c}472 \\
3,28 \\
\text { I } 4\end{array}$ \\
\hline Poids moyen à $\mathrm{I} 2$ semaines en $\mathrm{g} .$. & r 86 & I 186 & 1270 & 1316 & $\operatorname{II} 70^{\circ}$ & II $4 \mathrm{r}$ \\
\hline Mortalité cumuléé à I 2 sem. p. 100 & $\mathrm{I}_{4}$ & 8 & 6 & 6 & 24 & I4 \\
\hline
\end{tabular}

Dans les présentes conditions expérimentales, et en considérant la sévérité des épreuves, si les divers traitements assurent une protection suffisante contre la coccidiose caecale du poulet, il apparait que les meilleurs résultats soient obtenus avec 1'association furazolidone/nitrofurazone $50 / 50$ ou avec la furazolidone seule à $100 \mathrm{~g} /$ tonne. Dans tous les cas, s'installe une Immunité égale ou supérieure à l'immunité naturelle.

En outre, dans le cas des lots $3\left(\mathrm{CFZ}_{50} / 5 \mathrm{O}\right)$ et 4 ( $\mathrm{CF}$ IOo), les traitements permettent une croissance et des taux de conversion alimentaire qui les rendent supérieurs à tous les autres lots inoculés ou non.

\section{REMERCIEMENTS}

Nous tenons à remercier la Lyonnaise Industrielle Pharmaceutique et 1'Institut Professionnel de Contrôle et de Recherches Scientifiques des Industries de 1'Alimentation Animale pour la contribution matérielle qu'ils ont apportée à la réalisation de ce travail.

\section{SUMMARY}

300 broilers experimentally infected with caecal coccidiosis were treated with coccidiostatic associations furazolidone-nitrofurazone in doses of $8 / 55$, $\mathrm{I}_{5} / 50$ and $50 / 50 \mathrm{p} . \mathrm{p} . \mathrm{m}$. of feed respectively. The association 50/50 p. p. m. was shown to be superior to the others by its doccidiostatic activity, by the growth improvement and by the lowering of the consumption indices. Furazolidone used alone at roo p. p. m., has a lesser anticoccidial activity but gives greater gains in weight. All the treatments allow an anticoccidial immunity equal or superior to natural immunity.

I.N.R.A.

BIBLIOTHEQUE UO 3590 gocteur-Gérant: B. LACLAVIÈRE.

DOMAINE DE CROUELL:

63039

Clermant-FD Ceaex? 